\title{
Information flow in group exams
}

\author{
Steven F. Wolf, ${ }^{1}$ Timothy M. Sault, ${ }^{1}$ and Hunter G. Close $^{2}$ \\ ${ }^{1}$ Department of Physics, East Carolina University, Greenville, NC 27858 \\ ${ }^{2}$ Department of Physics, Texas State University, San Marcos, TX 78666
}

\begin{abstract}
Collaboration is an integral part of science, and as our classrooms become more collaborative, so too can our assessments. Group exam data gives us a new kind of data about how our students relate to each other. Network analysis provides many tools for describing, visualizing, and analyzing student networks. In particular, we have used Directed networks to describe student collaboration, explored relationships between measures of network position on exam performance, and examined what the different micro-structures tell us about the different kinds of collaboration on an exam. Furthermore, we describe trends in these collaborative practices over a semester-long time-scale.
\end{abstract}

\section{INTRODUCTION}

Collaborative classrooms are becoming the new normal. As a part of that transition, many instructors are using collaborative exams in addition to individual exams [1-6]. The PER community has long used assessments as a way of learning about our students. However, this has been generally limited to content knowledge or attitudes about science. Group assessments can give us insight into how our students choose to associate with one another. PER has a long history of studying the ways that students associate with each other [7]. By looking at who they collaborate with on group assessments, we can build a bridge between the cognitive and the relational.

At East Carolina University and Texas State University, some instructors have been giving two-phase exams in our introductory calculus-based physics courses, where the same exam is taken twice - first individually, then as a group [1]. A salient feature of this implementation is the use of open collaboration. In particular, students choose their collaborators and may switch between multiple groups during the course of the exam. Previously [1], we have used network analysis tools to describe the groups that students form in open collaboration settings. This showed that student collaboration behaviors are influenced by things such as the furniture in a room. Specifically, rooms with large round tables led to larger groups being created than tiered classrooms with individual movable desks [1]. Additionally, the group behaviors of students working in an open-collaboration environment were compared using metrics created by Beatty to the group behaviors of students in pre-determined groups [2].

We are interested in multiple properties of student collaboration networks. First, we will describe the classroom collaborative network, especially focusing on the different ways that students connect themselves to each other relative to the class. Another important area to understand is how grade interacts with the ways that students choose to acknowledge working with each other. To better understand these questions, we will adapt our analysis method to include the directionality of ties between students. We will discuss the different properties of asymmetric ties and mutual ties, especially looking at how these ties are related to exam perfor- mance. We will also explore the different ways that student position in the network is related to exam performance. Finally, we will explore each of these measures over the course of a semester to study how patterns in collaboration emerge and change.

\section{METHODS FOR ANALYZING GROUP EXAM NETWORKS}

Group exam collaboration networks can naturally be described using network analysis techniques. Network analysis employs two types of networks, directed and undirected - both types of network are used to examine social networks. For example, the analysis of collaborations between PER scholars utilized undirected networks as they were interested in how connections changed over time, and not the relative status of those connected [8]. However, during the span of a semester, students often choose to collaborate, or acknowledge collaboration, with other students based on perceived status, making directed networks an appropriate tool to describe these networks in more detail. Directed networks are created by drawing edges between different actors in the network with arrowheads [9]. So if student A says she worked with student B, an edge is drawn from A to B. If student B says she worked with student A, another opposite edge is drawn.

The field of network analysis has many tools for measuring status within a network. These are all loosely called centrality measures. The simplest centrality measure is degree centrality, the number of edges linked to any given node. Directed networks allow us to study outdegree centralitythe number of edges flowing away from the node-and indegree centrality-the number of edges flowing into a nodeseparately, as each indicates different types of imporance [9]. In our context, a student's outdegree is the number of collaborators nominated by the student, and the indegree is the number of students who identified that student as a collaborator.

While degree centrality is one way that we can measure status within a network, it is limited to the local structure around a particular actor. A more global measure is betweenness 
(a)

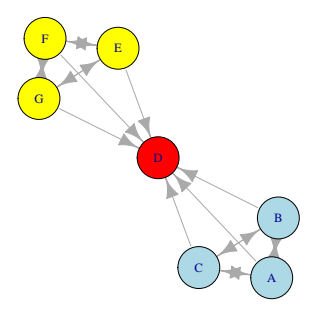

(b)

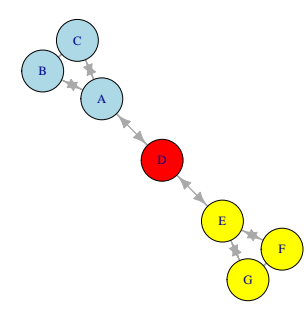

FIG. 1. (Color Online) Illustration of differences in the different types of centrality. In each figure, focus on the red node labeled D. All other connections in both graphs not related to node D are identical. (a) Node D has high degree and zero betweenness. (b) Node $\mathrm{D}$ has low degree and high betweenness.

centrality [9]. The betweenness centrality $C_{B}$ is "(roughly) defined by the number of geodesics (shortest paths) going through a vertex or an edge" [10]. The technical definition is:

$$
C_{B}(k)=\sum_{i, j} \frac{\partial_{i k j}}{\partial_{i j}}, \quad i \neq j \neq k
$$

where $\partial_{i k j}$ is the number of paths that flow from $i$ to $j$ through node $k$, and $\partial_{i j}$ is the number of paths that flow from $i$ to $j$. A path is not a random walk in the network. In a path, a single node may be traversed only once. An actor with a large betweenness centrality score is sometimes referred to as a "cut-point" due to the fact that if that actor were removed, the network would change in structure greatly. It is important to note that betweenness centrality is sensitive to tie direction.

In Figure 1(a), node D has a high indegree, but zero outdegree. Node D's betweenness centrality will be zero since there are no paths that flow through that node. That node is called a 'sink' because as it does not provide a path for nodes A, B, and C (the blue group) to share information with nodes E, F, and G (the yellow group). In contrast, Figure 1(b) illustrates a node which has relatively low degree, but high betweenness. Arguably, node B in Figure 1(b) has a more important position in the network because it fufills a betweengroup broker role, allowing information to flow back-andforth between the blue and yellow groups.

Given an open collaboration system, initial networks will be based on things like who you know or who you sat next to on the first day of class. So connections formed will not be based on prior knowledge of their classmates' physics skill. But if the class is building a community that values having and sharing physics knowledge, the exam network will develop structures that allow knowledge to be transfered between students and groups. Of the centrality measures that we mentioned previously, betweenness will be most sensitive to this developing community. Therefore we hypothesize that late semester exam networks will have higher betweenness scores than early semester exam networks of the same size.

Ties between nodes can be classified in three ways: Mutual ties - where the relationship is acknolwedged by both actors, asymmetric ties - where relationship is acknowledged by only one actor, and null ties - where no relationship is acknowledged. In networks describing well-developed communities, mutual ties are an indication of trust and deeper collaboration, while asymmetric ties in these networks are often an indication of differences in perceived status [9]. A dyad census reveals the number of mutual, asymmetric, and null (nonexistent) ties in a network, giving us insight into the level of reciprocity within the community. In this way, the type of tie between nodes is a way of indicating relative status between these two nodes. Status in a classroom is often tied to students' exam grades. Therefore, there should be a larger score difference in asymmetric dyads than there should be in mutual dyads on exams late in the semester.

By examining student group exam networks from two semesters using the above centrality measures and analyzing dyad properties, we will explore the following questions:

1. Do exam networks exhibit the properties of networks describing well-developed communities?

2. Is exam performance a proxy for network status?

\section{RESULTS}

\section{A. Sampling and procedure}

Students in our study taking group exams recorded their collaboration networks as described previously [1], by recording who they worked with mostly and sometimes on their exam answer sheet. While some students marked that they "sometimes" worked with others, this response was infrequently chosen, and we therefore created our exam networks by considering only the "mostly" networks. This analysis will focus on two classes which occurred in two semesters (Fall 2015, "Physics 1" and Spring 2016, "Physics 2 "). Both courses had 4 exams (3 midterm exams and 1 final exam) given in the duplicate exam format. Students who did not take all of the exams were removed from the sample, leaving $N=44$ students in the Fall semester and $N=36$ students in the Spring semester. It should also be noted that some students $(N=22)$ were in both classes. Therefore, many students in the Spring semester were "experienced" group exam takers from the beginning, while no Fall semester students had taken group exams in this format before. In the analysis below, we discuss correlations between network position statistics and exam scores, as well as engage in a microanalysis of pairs of students. All analyses were carried out in the statistical programming language R[11], using packages specifically designed for describing networks $[10,12]$.

Given the two-phase exam design, exams in this course were designed to be more difficult than in an average course. In general, the average score on the solo-phase exam was intended to be between $50 \%$ and $65 \%$, and the average group exam score was intended to be above $75 \%$. This pattern was followed for all exams except exam 3 in Fall 2015. Both the individual and group exams for Exam 3 were excluded from analysis because the exam was poorly constructed, Lastly, we 
will note that in these semesters, the two-phase exam was combined with a take-home portion for exams 1-3. So for these early exams, the number of questions was relatively low $(\approx 10-15)$ and the distribution of scores is non-parametric.

\section{B. Exam networks as social networks}

As betweenness is a measure that takes the graphs global structure into account, the betweenness statistics should be more sensitive to higher-order connectivity in the class. Figure 2 displays the Empirical Cumulative Distribution Function (ECDF) for the betweenness statistic for the Fall and Spring semesters. In other words, it is a cumulative probability distribution function. The value $\operatorname{ECDF}(x)$ is the probability that a random value drawn from a given distribution is $\leq x$. The Fall semester exam 1 distribution is significantly shifted to the left as compared to the exam $2 \& 4$ distributions, indicating overall lower betweeness in exam 1 . We compared the betweenness centrality distributions for each semester using a two-sided Kolmogorov-Smirnov test (KStest), appropriate here due to the fact that these measures are non-parametric. The KS-test statistic for two empirical distributions is the maximum vertical distance between the ECDFs of those distributions. The null hypothesis for each KS test is that the distributions are the same, and we are testing at a 95\% confidence level. In the Fall, we found betweenness scores were significantly lower on exam 1 than they were on exam $2(p=0.006)$. Also in the Fall, we found betweenness scores were significantly lower on exam 1 than they were on exam $4(p=0.003)$. Comparisons between later exams in the Fall (exams 2 and 4) do not reject the null hypothesis. The fact that the betweenness distributions increased supports the idea that the exam networks exhibited more properties of well-developed communities as the semester progressed.

As the Fall and Spring semesters had different numbers of students, betweenness distributions cannot be directly compared. However, comparisons within the Spring semester exams can still be made. Given the fact that nearly two-thirds of the Spring class had taken the Fall class with group exams, we expect that part of the social network structure developed in the Fall may be intact from day 1 in the Spring. So the increase in the betweenness distribution observed in the Fall is not expected in the Spring. In fact all comparisions among betweenness distributions do not reject the hypothesis that they are the same for all pairs of exams, with the exception of exams 2 and $4(p=0.018)$. By visually inspecting the distributions (see Figure 2), we note that the difference is most pronounced at higher betweenness scores. It is difficult to make any conclusions about why this might be. Many factors, including exam timing and content, could be at play. More study is clearly needed in this regard. (a)

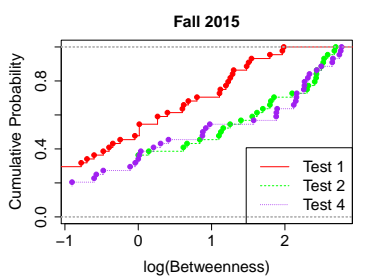

(b)

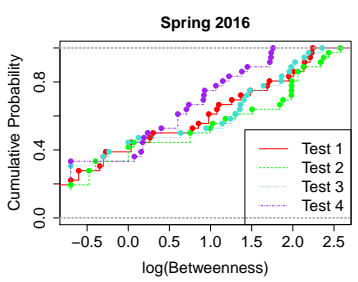

FIG. 2. (Color Online) Cumulative probability of $\log$ (betweenness) scores for (a) Fall 2015 and (b) Spring 2016 semester. Note that since some of the nodes have a betweenness of zero, the plot does not start with a y-value of zero due to the log scale.

\section{Exam performance and status}

Lastly, we performed an analysis of all dyads (pairs of nodes) in each graph. Specifically, we examined differences between asymmetric ties and mutual ties related to individual exam grade. Our goal in allowing open collaboration is to promote the development of a well-connected learning community. This is not to say that status differences will go away over the course of student interactions. In fact, we still note that there were a significant number of asymmetric ties in the Spring compared to the Fall. Instead, we ask if students can identify students of higher status and use that knowledge to their benefit. Therefore, we compared the grade differential on the individual exam of students in mutual vs. asymmetric ties. When student networks are observed early in the semester, we predict that the grade differential will be the same for students in mutual vs. asymmetric dyads as students haven't gotten to know the physics skills of their classmates yet. However, as this knowledge of their peers develops throughout the semester, we predict that score differential in asymmetric dyads will be higher than score differential in mutual dyads. For this comparison, a mutual tie is the same as two asymmetric ties between the same set of nodes. For each pair of linked students, we calculated the score differential as:

$$
\operatorname{diff}=S_{\mathrm{To}}-S_{\text {From }}
$$

where the $S_{\mathrm{To}}$ is the score of the person who was mentioned as a collaborator, and $S_{\text {From }}$ is the score of the person who mentioned the collaboration. By this definition, the distribution of score differences for mutual ties must be both symmetric and have an average of zero. On exams 1-3 in both the Fall and Spring semesters, we found no significant difference the grade difference distributions for mutual and asymmetric ties using a KS-test at a $95 \%$ confidence interval. However, on exam 4 (the final) for both semesters, a KS-test found significant differences ( $p=0.004$ in the Fall, and $p=0.0009$ in the Spring) between the score distributions. In particular, lower-scoring students tended to nominate their higherscoring peers more often in asymmetric relationships. 
(a)

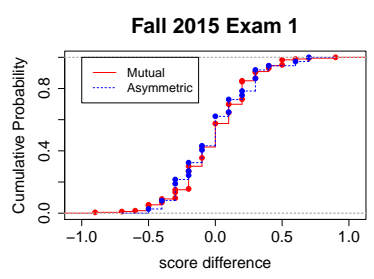

(b)

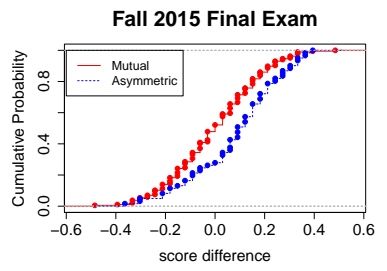

(c)

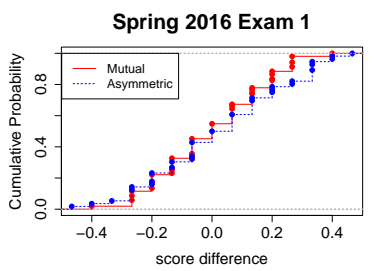

(d)

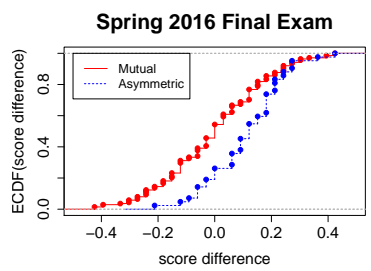

FIG. 3. (Color Online) ECDFs of grade differential for students in mutual and asymmetric ties for Fall 2015 (a) Exam 1 and (b) Exam 4 and Spring 2016 (c) Exam 1 and (d) Exam 4. Only on both final exams do we find a significant difference between exam grade differentials. Since the blue curve is shifted to the right in (b) and (d), we observe that the score differential is larger for asymmetric ties than mutual ties.

\section{CONCLUSION}

We explored the collaboration behavior of students in introductory physics classes in Fall 2015 and Spring 2016 on group exams administered in a open-collaboration environment. We found that students became better collaborators as time progressed as the network became more connected and students used information about status to their advantage. One of the explicit goals of the instructor in these courses is to develop community that expands beyond the term of the semester, and pushes people outside of their initial peergroups. Open collaboration exams are an important step towards reaching this goal.

Given our community building goal, we studied how each student's placement within the network becomes more central to the network. As betweenness centrality is a measure that takes the graphs global structure into account, we explored how betweenness distributions change over the course of the semester. We found that betweenness increased during the Fall semester, and held stable during the Spring semester (when many of the students already had an established network due to their experience with the instructor from the Fall). This supports the idea that the exam networks exhibited more social network properties as the semester and academic year progressed.

Lastly, we discussed the different ways that pairs of students interacted, noting whether ties between these pairs were mutual or asymmetric. On all exams, except the final exam each semester, there were no significant difference between the score differentials of actors with mutual and asymmetric ties. On the final exams, however, asymmetric ties were more likely to go from low scoring to high scoring students. This suggests that actors are becoming self-aware of their grade in the class, and seeking help as needed. It may indicate that students who share knowledge, do so freely, without making a specific note of it and that students who seek knowledge are more likely to acknowledge the source.

In the future, we will look to build on our dyad analysis results by looking at triad behavior. Transitivity-a property of certain kinds of triads - is another tool for describing community development and relative status within that community. We plan to explore how transitivity relates to global network position measures, as well as exam performance. We will also study the different kinds of roles students play in the network using techniques common in the social network analysis field, such as block modeling and regular equivalence. Additionally, we plan on interviewing students taking group exams to explore their perceptions of the group exam process and to describe the ways that they choose to indicate collaboration, potentially leading to the collection of multi-relational data.
[1] S. Wolf, C. Blakeney, and H. Close, in Physics Education Research Conference 2016 (Sacramento, CA, 2016), PER Conference, pp. 400-403.

[2] I. Beatty, in Physics Education Research Conference 2015 (College Park, MD, 2015), PER Conference, pp. 59-62.

[3] Y. Lin and D. Brookes, AIP Conference Proceedings 1513, 254 (2013).

[4] C. E. Wieman, G. W. Rieger, and C. E. Heiner, The Physics Teacher 52 (2014).

[5] B. H. Gilley and B. Clarkston, Journal of College Science Teaching 43, 83 (2014), ISSN 0047231X.

[6] H. Leight, C. Saunders, R. Calkins, and M. Withers, Cell Biology Education 11, 392 (2012), ISSN 1931-7913.
[7] E. Brewe, L. Kramer, and V. Sawtelle, Physical Review Special Topics - Physics Education Research 8, 010101 (2012).

[8] K. A. Anderson, M. Crespi, and E. C. Sayre, Phys. Rev. Phys. Educ. Res. 13, 010121 (2017).

[9] C. Prell, Social Network Analysis: History, Theory and Methodology (SAGE Publications, 2011), ISBN 9781412947152.

[10] G. Csardi and T. Nepusz, InterJournal Complex Systems, 1695 (2006).

[11] R Core Team, $R:$ A Language and Environment for Statistical Computing, R Foundation for Statistical Computing, Vienna, Austria (2015).

[12] C. T. Butts, sna: Tools for Social Network Analysis (2016), R package version 2.4. 